\title{
Desafíos de las cajas de compensación familiar frente a las nuevas tendencias del mercado
}

\author{
Challenges of family compensation funds in the face of new market trends
}

DOI: 10.21803/adgnosis.v8i8.365

\section{Resumen}

Tomando en cuenta los retos y desafíos actuales a que se enfrentan las cajas de compensación familiar, se pretende plantear bases sólidas para generar estrategias en pos de atraer más clientes estas debido a los nuevos retos del mercado, que con su constante cambio generan una dinámica totalmente nueva en todos los segmentos del mismo. Es así como, a través de una revisión bibliográfica una entrevista no estructurada, este artículo de investigación hace referencia al marketing relacional, el marketing emocional, y la gerencia del servicio que entran a formar parte de las estrategias para este tipo de empresas desde el punto de vista de su objeto social y de desarrollo mercadológico. Es así como en los resultados principales se plantean los retos y desafíos para estas organizaciones como publicidad, Marketing directo, Marketing promocional. Animación, Realización de eventos y Comunicación digital.

Palabras clave: Cajas de Compensación Familiar, Marketing Relacional, Marketing Emocional, Gerencia del Servicio, Estrategia, Posicionamiento.

\section{Abstract}

Taking into account the current challenges faced by the family compensation funds, the aim is to establish solid foundations and generate strategies to attract more clients due to the new challenges of the market, which its constant change generates a total new dynamic in all market segments. Thus, through a bibliographic review of an unstructured interview, this research article refers to relational marketing, emotional marketing and service management which are part of the strategies for this type of business from the point of view of its social purpose and market development. This is how the main results present the challenges for these organizations such as advertising, direct marketing, promotional marketing. Animation, Event Production and Digital Communication.

Keywords: Family Compensation Funds, Relationship Marketing, Emotional Marketing, Service Management, Strategy, Positioning.

\author{
Rubén Cubillos Coll \\ María Cristina Rosales Orozco
}

Lina Jaramillo Alzate

jaramillolina1@gmail.com

\section{Cómo citar este artículo:}

Cubillos R., Rosales M. \& Jaramillo L. (2019). Desafíos de las cajas de compensación familiar frente a las nuevas tendencias del mercado. 8(8), pag.129-140 DOI: 10.21803/adgnosis.v8i8.365 


\section{Introducción}

Las Cajas de Compensación Familiar han sido reconocidas como un operador social eficiente para suministrar servicios sociales no sólo a su población afiliada, sino también a la población en vulnerabilidad no afiliada, por tanto, su competencia y experiencia les permite proponer y operar en forma eficiente programas sociales, comenzó como una remuneración adicional que brindaban voluntariamente las empresas a los trabajadores con familia (personas a cargo), preocupados por su situación precaria; hasta convertirse en una obligación reglamentada por la ley, propuesta con el fin de atenuar los problemas sociales existentes en los países.

El riesgo permanente de los cambios de ley en muchos países, mantiene en expectativa a las Cajas de Compensación haciéndolas organizaciones más competitivas y que trabajen por ser autosostenibles, a través de la venta de servicios a las empresas afiliadas para sus trabajadores, la venta de estos a nivel masivo con el uno a uno (one to one) y de esta manera es posible no depender únicamente de los aportes parafiscal para cada país, sino que a través de la oferta de sus servicios se alcancen los ingresos suficientes para superar las crisis de este tipo.

Es de esta manera como se hace necesaria la innovación en el Portafolio de Servicios de las Cajas para brindar ofertas atractivas y novedosas al mercado empresarial, entregando servicios que respondan a las necesidades de los sectores, este conocimiento se logra investigando en el mercado empresarial cuales son las expectativas específicas y de esta manera lograr brindar alternativas a la medida de las necesidades de este segmento objetivo.

Los cambios permanentes del mercado y las nuevas exigencias de los consumidores hacen que las empresas como las cajas de Compensación Familiar, se proyecte en el tiempo a través de ofertas innovadoras. Es así como basado en información actualizada de los servicios que contratan las grandes empresas afiliadas a las cajas de compensación, la elección, frecuencia de uso y las ofertas poco atractivas para las empresas, se propone el diseño de estrategias, en busca de mejoras e innovación en el portafolio, que permitan aumentar la demanda de los servicios.

Bajo este contexto, se pretende analizar a por medio de las tendencias de marketing más actualizadas, los desafíos a los cuales se enfrentan las cajas de compensación familiar de cara al futuro. Para tales fines se inicia con un apartado teórico en donde se exponen los modelos de marketing actuales que servirán como base para plantear dichos desafíos, seguidos de la metodología en donde se especifican los aspectos requeridos para llevar a cabo la presente investigación. Posteriormente los hallazgos se presentan planteando el marketing de contenidos y la gerencia de servicio como los desafíos para las cajas de compensación y finalmente realizando algunas conclusiones respecto a estos retos.

\section{Aproximación Teórica}

\section{Marketing de contenidos}

El marketing de contenidos ayuda a entender lo que quieren y esperan los usuarios, sus necesidades, nuestro público objetivo y nuestro target. Gracias al marketing de contenidos descubrimos cómo debemos mostrárselo a nuestro público. Los formatos, las plataformas, en qué medida, etc., para que nuestro contenido les resulte interesante y, sobre todo, útil y de valor.

Una técnica de marketing de creación y distribución de contenido relevante y valioso para atraer, adquirir y llamar la atención de un público objetivo bien definido, con el objetivo de impulsarles a ser futuros clientes. Para las cajas, el contenido es de relevancia, ya que de una manera clara y sencilla deben llegar a sus futuros afiliados a través de los medios. El contenido expresa el sentir de las cajas para sus afiliados y las estrategias que se podrían seguir son las siguientes; Con tu blog o cualquier estrategia de marketing digital puedes ayudarle a informarse y a crearse una opinión sobre un tema determinado; Con tus e-books y whitepapers puedes ofrecerle información comparativa de 
distintas soluciones a su problema o necesidad. En la página de precios o de servicios puedes demostrarle por qué tú eres su mejor opción. A través de vídeos con entrevistas a testimoniales puedes transmitirle lo positivas que han sido las experiencias de otros clientes con la marca.

Así mismo, estos son algunos tipos de contenidos que puedes utilizar en tu estrategia de content marketing; Imágenes; Infografías; Vídeos; Guías/tutoriales; Revistas; E-books; Plantillas; Whitepapers; Presentaciones; Aplicaciones; Juegos y Webinars.

\section{Marketing Relacional}

El IMC (por sus siglas en inglés) se refiere a las comunicaciones integradas de marketing. (Integrade marketing communications), es un sistema cuyo objetivo es integrar toda la cadena productiva y comunicarla a través del mix promocional.

Los mercados industriales distan mucho de los mercados de consumo. Lo ideal es crear un mix efectivo, que al mismo tiempo permita crear un IMC eficiente. La Asociación Americana de Agencias de Publicidad define el IMC como un concepto que reconoce el valor añadido de un plan integral que evalúa las funciones estratégicas de una variedad de disciplinas de la comunicación, y combina estas disciplinas para proporcionar claridad, coherencia y un máximo impacto de la comunicación.

El IMC no es sólo el uso de muchos canales, se trata de hacer todas las acciones de marketing juntas bajo una misma idea con el fin hacer crecer una empresa y que el comportamiento de la misma sea coherente. Las campañas de marketing integrado combinan las herramientas y tácticas del marketing online y tradicional para conectar con los consumidores cruzando las plataformas. La meta de IMC es integrar el mensaje de la marca con los distintos canales e incrementar las ventas y la confianza de la marca obteniendo los beneficios esperados. Teniendo en cuenta lo anterior, abordar el criterio de marketing relacional exige criterios rigurosos que establezcan sus verdaderas aportaciones con respecto a la doctrina general del marketing.
El consumidor actual se enfrenta a diario a una infinidad de mensajes orientados a provocar en él un efecto determinado. Estos mensajes, claramente construidos como publicitarios o disfrazados de información, persiguen al moderno consumidor en todas y cada una de sus facetas diarias: laborales, familiares, sociales y, por supuesto, personales. La televisión, la radio, el cine, los medios impresos, el correo, el ordenador, el teléfono e incluso el boca a oreja, son las bases desde las que las marcas tienden a construir los mensajes con los que pretenden llegar a ese inmenso colectivo que formamos los consumidores.

Sin embargo, las utilizaciones de cada vez más medios para poder llegar hasta los sujetos están provocando el efecto contrario al deseado por las marcas; $y$ es que, ante tal exceso de diferentes mensajes, el consumidor comienza a ser escéptico y no sólo no asimila, sino que cuestiona, la validez de los mensajes que recibe (Reinares \& Fernández, 2003).

Para el caso de las cajas de compensación, se es claro, que no pueden seguir trabajando bajo el amparo del marketing tradicional sino quieren deja de existir en el mercado, es por ello, que una buena base de marketing relacional estará diseñada a la medida de ellas, teniendo en cuenta los siguientes parámetros: El marketing relacional puede definirse como una estrategia de negocio o actividad que persigue la captura de un mayor "share of wallet" utilizando datos sobre clientes y el análisis de los mismos, con trasparencia en el razonamiento económico, y con el objetivo de adquirir, retener y desarrollar a los clientes rentables de una forma aún más rentable.

Para efectos de este artículo de investigación, el share of wallet, la cuota de cartera, que es como se conoce al share of wallet en español, ayuda a decidir si asignar recursos para ampliar la categoría de productos, conquistar clientes de otros competidores o incrementar la frecuencia de compra. Las cajas de compensación deben tener un objetivo claro con respecto a sus segmentos y donde invertir sobre la rentabilidad de los clientes que ya tiene y los potenciales. De esta manera es conveniente el marketing relacional para; Integrar todas las funciones de la caja; Uso expandido 
de tecnologías de la información. (Facebook, WhatsApp, Instagram, entre otros.); Visión global. Perspectiva de visión empresarial; Alianzas estratégicas y cooperación con marketing. Con los proveedores de la caja; Relaciones de largo plazo. Retención de afiliados; y Socios. En vez de afiliados, se trata de hacer a cada cliente (o pequeños grupos de clientes) actual o potencial la oferta que consiga maximizar su valor (en términos de tasa de éxito, ingresos, coste y duración), mediante la generación y explotación eficaz de la información que existe sobre él en la empresa.

El punto central de este tipo de marketing consiste en retener clientes y venderles nuevos más rentables productos (servicios) muy adaptados a sus necesidades (cross-selling y up-selling).

Y es que, desde el punto de vista de los costes de la empresa suele resultar más rentable retener a los clientes existentes que empeñarse en captar clientes nuevos. El camino para retenerles es tenerles siempre satisfechos, ofreciendo en todo momento productos cada vez más a su medida que cubran sus necesidades, y para ello es preciso disponer de gran des cantidades de información. En resumen, los consumidores dejan de ser clientes para convertirse casi en auténticos socios de cada compañía (Reinares \& Fernández, 2003).

Es así como las cajas de compensación deben realizar esfuerzos para relacionarse con sus afiliados a través de las siguientes formas de comunicación; $\mathrm{Pu}-$ blicidad. A través de estrategias ATL y BTL, así mismo a través del marketing digital, esto con el fin de hacer marketing de atracción conseguir nuevos clientes; Marketing directo. One to one, para hacer relación de fidelización con los afiliados y sus familias; Marketing promocional. Acorde con el plan publicitario de comunicaciones de la empresa a través del IMC al que se refirió anteriormente; Animación. Consolidando estrategias de merchandising en las distintas sedes de las cajas; Realización de eventos. En algunos casos, los eventos serán patrocinados por la misma caja para buscar aumentar la fidelización de los afiliados existentes y atraer a los potenciales; Comunicación digital. A través de sus páginas web (que deben estar constantemente actualizadas), Twitter, Facebook,
Instagram, YouTube, entre otros.

Además, debe contar con las siguientes herramientas de marketing; Minería de datos: Un database management, el cual debe estaré actualizado con respecto a sus afiliados y esta gestión de datos e información debe ser respaldada por un sistema de seguridad fiable. Esto conlleva a realizar una gestión de modelos predictivos para prospectar; Análisis clúster: En caso de que esta estrategia sea prioridad para las cajas, es un buen recurso para que posteriormente se presten los servicios demandados por los afiliados y sus familias. Se hace énfasis en la segmentación y microsegmentación, que deben ser propias de las cajas de compensación; Call center. La plataforma de gestión telefónica de cada caja. Esto no debe ser una tendencia de moda, sino una prioridad de la gerencia del servicio, tema que trataremos más adelante.

El call center es un sistema de relación con los afiliados a través de la voz y no a través de máquinas contestadoras. En primera instancia, el Fulfillment, el cual, Teniendo en cuenta la importancia del manejo de la información, sobre todo el tema de habeas data. En este sentido los empresarios tienen la opción de externalizar el proceso de Fulfillment y distribución o crear un departamento interno que se encargue de este proceso.

En segunda instancia el análisis de resultados, dado que una vez se realicen todas las actividades, se analizan todos los ítems para verificar los resultados a través de Kpis. La palabra KPI proviene de "Key Performance Indicators". Esto se traduce, ni más ni menos, como los "indicadores claves de desempeño”. Es decir, aquellas variables, factores, unidades de medida, que consideramos "estratégicas" en nuestra empresa. Y que por ende influyen directamente en el “core business". Los KPI'S son métricas (medibles y cuantificables) que determinaran numéricamente una variable (por ejemplo: ingresos, gastos, número de visitas...) directamente relacionada con los objetivos marcados dentro de nuestra estrategia o plan de marketing anual.

El establecimiento de KPI'S en una empresa sue- 
le derivar generalmente en un Cuadro de Mandos o Balance Score Card, herramienta que recogería los principales indicadores de marketing o de la empresa a nivel gerencial/presidencia.

Todo esto se refleja en el tema principal de segmentos y microsegmentos, quienes finalmente son la razón de ser de las cajas, esto incluye a los clientes internos y a los stakeholders, quienes son todas aquellas personas, grupos y entidades que tienen intereses de cualquier tipo en una empresa y se ven afectados por sus actividades. Son interesados, directos $\mathrm{o}$ indirectos, en que la empresa funcione ya que en caso contrario les afectaría directamente.

Finalmente, para este tema de marketing relacional, se exponen 12 claves para que las cajas actúen en concordancia con sus afiliados. Esto, entendiendo que no todos los afiliados son iguales, y teniendo en cuenta que los segmentos son diferentes.

Según Farné (2010) los segmentos se clasifican de la siguiente manera:

1. Dependientes.

2. Pensionados.

3. Voluntarios.

4. Facultativos.

\section{Afiliados por fidelidad.}

Dada su misión vocacional, las Cajas de Compensación Familiar se han consolidado como gestores sociales y como una extensión de la responsabilidad social empresarial de sus empresas afiliadas. Es por esto, que las estrategias de marketing social tienen alta relevancia.

\section{Marketing emocional}

En las 22 leyes inmutables de marketing (Ries \& Trout, 1993) hacen énfasis en la mente, antes de que se hablara del neuromarketing, es mejor ser el prime- ro en la mente que el primero en el punto de venta. Un producto que tiene presencia en la mente de los consumidores debe ser preferido ante un producto del cual no se tiene ningún tipo de información, aunque haya llegado primero a la tienda. Si el punto clave seria llegar primero a la tienda no existiría la promoción, los clientes buscan satisfacer sus necesidades con alternativas antes conocidas y las cuales han sido percibidas por su mente.

La ley de la mente es una consecuencia de la ley de la percepción. Si el marketing es una batalla de percepciones, no de productos, entonces la mente tiene prioridad sobre el punto de venta.

Para las cajas de compensación es prioritario estar en la mente de sus afiliados. El top of mind y el top of hearth, son las variables que finalmente desde los postulados del marketing hacen la diferencia para posicionarse en su target. A través de lo emocional, vale la pena rescatar las nuevas tendencias principales del marketing: De un marketing de producir y vender a uno de sentiry responder.

Asimismo, de poseer activos a poseer marcas; De una integración vertical a una integración virtual; De un marketing masivo a un marketing personalizado; De operar en el mercado a operar en el ciber espacio; De tratar de lograr participación en el mercado a tratar de lograr participación del cliente; De enfocarse en atraer clientes a enfocarse en retener clientes; De un marketing de transacciones a un marketing de relaciones; De la adquisición del cliente a la retención y satisfacción del mismo; De un marketing de intermediarios a uno directo; De un monólogo de vendedor a un diálogo con el cliente; De un plan de comunicación aislado a comunicaciones IMC; De marketing se un solo canal a un multicanal; De marketing centrado en el producto a un marketing centrado en el cliente; De un departamento de que lleva cabo el marketing a una compañía en donde todos hacen marketing; De explotar proveedores y distribuidores a colaborar con ellos (Kotler, 2005).

De todos los postulados de marketing esbozado por Kotler (2005) se rescata el marketing de producir 
y vender a uno de sentir y responder, de un marketing masivo a un marketing personalizado, de tratar de lograr participación en el mercado a tratar de lograr participación del cliente, de un marketing de transacciones a un marketing de relaciones, de un monólogo de vendedor a un diálogo con el cliente.

Es así, como se resalta, el sentir y responder. Debemos llegar al corazón del afiliado a través de las emociones que le producen la prestación de los servicios de la misma, es así, como, a través de los segmentos debemos personalizar a cada uno de los afiliados y conocerlos mejor, así cada uno de los afiliados es un punto de fidelización y por ende de participación en la rentabilidad de la caja en la medida en que la utilice. Se resalta nuevamente el marketing de relaciones y las personas encargadas de la labor de ventas, no será solo de un vendedor, sino de una migo y asesor del afiliado.

Así pues, el marketing emocional es la disciplina del marketing que utiliza una marca dentro de una estrategia, con el objetivo de lograr un vínculo afectivo con usuarios, consumidores, clientes y futuros clientes, para que estos sientan la marca como algo propioy necesiten ser parte de ella. Pero en una sociedad en la que los usuarios cada día son más exigentes, el trabajo de las marcas es, en consecuencia, cada día más complicado. Además, no olvidemos que estamos en la era digital, donde la inmediatez está a la orden del día y, además, tenemos todos los recursos a nuestro alcance. Es decir, que no todo vale.

\section{Vínculo afectivo marca - consumidor}

Cabe resaltar que sin el vínculo afectivo marca-consumidor, el marketing emocional sería inexistente. Carecería de todo sentido. Esa relación o vínculo emocional que les haga vivir experiencias por ambas partes. Es decir, no solo el consumidor o cliente vive experiencias, también las marcas lo hacen a través de estos que, afortunadamente para las marcas inteligentes, sabrán sacarle provecho de ese feedback que el consumidor es capaz de dar.

Gracias a la comunicación bidireccional, marcas y consumidores se encuentran en el mismo nivel de co- municación, pero no nos olvidemos que es importante entender, para las marcas, que los consumidores quieren ser parte de ellas, quieren ser escuchados, tener voz para opinar sobre sus productos y servicios.

Por una parte, la marca genera un producto que cubre las necesidades de sus consumidores, y por otra, el consumidor y/o cliente está satisfecho, no solo por el producto porque cubre sus necesidades, sino por las emociones y sensaciones que este le produce. Eso es más experiencia de usuario.

En el caso de las cajas, ésta tiene un sentido de marca único, en donde se conjuga algo muy importante como lo es la región y/o ciudad, lo que le da un top de marca importante y se considera per se, la estrategia de marca de las mismas. Ejemplo de ello son los nombres como Combarranquilla, Cajacopi, Comfama, Comfenalco, cuyas siglas hacen eco en la mente de los consumidores.

Orozco (1999) comenta que el nombre y logo símbolo utilizado para la marca de una familia de productos en una empresa, constituyen la identificación visual que hace el consumidor en el proceso de compra. La decisión de buscar una identidad es parte de las acciones que se toman al crear empresas y la importancia que se le dé se justifica por el papel que jugará en el desempeño futuro de las ventas.

\section{Metodología}

El presente artículo se enmarca dentro del contexto de los estudios cualitativos, explicando descriptivamente la conducta de los sujetos involucrados en la investigación, partiendo de lo específico a lo general (inductivo), por lo tanto, es subjetiva e interpretativa, ya que toma en cuenta la naturaleza social e individual de los investigados, por ello, es el método de investigación social más relevante por excelencia (Oconnor, Zaldívar \& Hernández, 2011).

El Método Descriptivo se propone a través de la Observación participativa planteando una entrevista de respuestas abiertas (semiestructurada-) al director de la caja de compensación Combarranquilla, situada 
en la ciudad del mismo nombre en el norte de Colombia. En esta entrevista, el entrevistado posee la máxima libertad de respuesta, dentro de los límites de los tópicos de interés para el entrevistado (Aaker, Day \& Kumar, 2001). El diseño transversal, las variables se miden en una sola ocasión en el tiempo. Es así como desde los cualitativo, se observó y se midió en una sola ocasión la fuente primaria del estudio.

\section{Hallazgos}

Basado en los planteamientos anteriores acerca del marketing relacional y emocional, aparece la parte operativa del mismo que se traduce en la labor del marketing de servicios como factor competitivo de las cajas. Para ello, en la entrevista, el Dr. Ernesto Herrera Díaz Granados, director de la Caja de Compensación de Combarranquilla el día 2 de febrero de 2019, expresa que nuestra siguiente, esto acompañado con la excelencia del servicio a través de un sistema de gestión de calidad muy robusto que tenemos ya, con más de 15 años de venir trabajando en él, han permitido que nosotros seamos considerados y aceptados y evaluados en muy buena forma, la calidad de nuestro servicio y percibido de gran manera por nuestros afiliados, así que pensamos que debemos dar un paso más allá de la excelencia en el servicio a buscar lo que la realidad del mercado y lo que el cliente actual demanda, los usuarios de servicio demandan, lo que vemos que está haciendo y es la experiencia en el servicio, nosotros queremos que todo el que entre a Combarranquilla viva una experiencia excepcional de servicio que le permita regresar o que lo motive a regresar y que se vuelva un promotor de nuestros servicios y de nuestra Caja de compensación con sus allegados y con sus colegas de trabajo.

Es así como entra a la escena para las cajas de la gerencia del servicio, que, como tal, se encarga de las estrategias de servucción a través de todo su personal de marketing y ventas.

Se entiende por servucción como un neologismo que se emplea en el ámbito de la gestión empresarial para el proceso que se lleva a cabo a la hora de producir un servicio. "Detrás de todo gran servicio está la servucción”. La servucción es un elemento clave en la gerencia del servicio que lleva a que sus tres elementos sean básicos para afrontar los cambios prospectivos en el mercado.

Para definir la palabra servucción se toma en cuenta que es el proceso por medio del cual se produce un servicio. Siempre se piensa que la producción y servicio son dos temas diferentes y si lo son, pero por medio de la servucción analizamos que tienen una relación cerca y hasta se complementan. El servicio también se produce, se elabora, todos aquellos elementos físicos y humanos que intervienen en la relación que se da entre cliente y empresa. Cuando se habla del proceso de producir el servicio se refiere a la preparación del talento humano, equipos, infraestructura que influyen y tienen un impacto en la relación y el concepto que tendrá el cliente acerca de la empresa y el servicio que le prestaron.

Comose mencionó anteriormente en la servucción intervienen ciertos elementos que hacen posible este proceso como son; Los clientes quien es un elemento primordial y es quien recibe la fabricación del servicio; La parte física, es el material que permite la producción del servicio esta abarca toda la infraestructura de la empresa donde el cliente consume el servicio y todo aquel elemento físico que interfiera en él. El talento humano o personal de contacto, son las personas a disposición de la empresa quienes representan y son la imagen de la empresa y tienen el contacto directo con los clientes. Este aspecto es muy importante debido a que son una parte importante en la empresa porque son ellos quienes reciben, atienden y despiden a los clientes y su trato y atención influye en el concepto que el cliente se hace de la empresa, una mala atención podría ocasionar la pérdida de clientes.

El servicio, el elemento final el cual es el resultado de la interacción de los tres elementos anteriores, en este resultado se debe evidenciar un beneficio el cual satisfaga la necesidad que tiene nuestro cliente durante el proceso del servicio, es importante el entrenamiento previo para evitar caer en errores debido a que estos no se pueden solucionar durante el proceso y puede traer graves consecuencias a la empresa. 
En la servucción todos los elementos son importantes, puede existir el caso de encontrar una empresa con una infraestructura impecable, buenas instalaciones, pero al momento de evaluar su atención no es la mejor o un caso contrario, encontramos una empresa con una infraestructura no tan buena pero su atención y servicio pueden obtener una buena calificación. Teniendo en cuenta lo anterior, la total satisfacción del cliente debe ser el factor primordial para el sistema de servucción.

Es necesario cumplir con cada objetivo, a una empresa no le es factible ser excelente en un aspecto y mediocre en otro, debe existir un equilibrio y complemento en todos sus procesos. "Lo único que cuenta es un cliente satisfecho”. Jan Carlzon presidente de scandinavian Airlines. A partir de esta frase, la servucción termina en la satisfacción del cliente, que para este caso son los afiliados.

Zeithaml et al. (2001) indica que "un esquema de servicio es un cuadro o un mapa que retrata en forma precisa el sistema de servicio de modo que las distintas personas involucradas en proporcionarlo lo comprendan y traten con él, en forma objetiva sin tener en cuenta sus papeles o sus puntos de vista individuales” (p. 253).

Para una mejor comprensión de los términos a utilizar en el esquema de servicio, optaremos por presentar la definición dada por Zeithaml et al. (2001) para cada uno de estos; Evidencia Física; Acciones del cliente; Contacto en el escenario; Contacto tras bambalinas; Procesos de Apoyo; Línea de Interacción; Línea de Visibilidad; Línea de Interacción Interna.

Siguiendo los patrones del esquema del marketing de servicios, las cajas deben gestionar los procesos, o mejor, producir los servicios de la caja para una mejor prestación de los mismos.

Se debe tener un proceso interno de calidad, a través de una organización interna que se traduce en una excelente misión, visión, valores y principios en donde todos tengan una cultura organizacional que le de identidad a la caja (esta área no es visible a los afiliados).
A través de un soporte físico adecuado, traducido en maquinarias, equipos, materiales e insumos para el trabajo diario de los clientes internos y aunado al talento humano de alta competencia, nos mostramos como la cabeza visible de la caja (Esta área es visible para los afiliados).

Las interacciones se dan a través de lo visible y los clientes ofreciéndoles servicios que interactúan constantemente con ellos y hace del servicio el factor competitivo número uno del que hablamos anteriormente. Esto implica que todos los clientes internos de la caja son el factor visible de la misma, y el servicio de excelencia se da con el acompañamiento de la organización.

Por otro lado, están los retos de la administración y el marketing de servicios (Dirección - gerencia- CEO y SEO), tal y como lo expresa Zeithaml \& Bitel (2001) la estrategia se trabaja a través del triángulo del marketing de servicios.

\section{Marketing Interno (Posibilitando las promesas)}

Esta clasificación corresponde a todas las tareas que la empresa realiza internamente- con sus empleados en post de brindar un servicio de calidad y excelencia. En el caso específico de la idea emprendedora, se desarrollarán actividades tales como:

1. Capacitaciones al personal con el fin de fomentar las relaciones interpersonales y brindar las herramientas necesarias para una atención diferencial al público.

2. Programas de Motivación a corto y largo plazo. Con el fin de estimular, reconocer y premiar los objetivos alcanzados de cada empleado. Fomentar el sentido de pertenencia.

\section{MarketingExterno (Formulando las promesas)}

Esta clasificación corresponde a todas las tareas que la empresa realiza externamente- para sus clientes actuales y potenciales. La empresa realiza "promesas”. 


\section{Marketing Interactivo (Cumpliendo las pro- mesas)}

Esta clasificación corresponde a todas las tareas que la empresa realiza con sus clientes para efectivizar/cumplir con las promesas comerciales. Estrategias de cara al cliente interno en pos del cliente externo (Afiliado).

Es de esta forma como el entrevistado, con acierto, afirma que los retos están en la conquista de nuevos mercados, ya que a pesar de que no son muchas las cajas por ciudades, se establece competencia entre ellas para captar afiliados, que es con el nombre que se conocen los clientes de las mismas. Por ende, el segmento definido para ellas son los trabajadores dependientes, independientes, y pensionados en general, quienes laboran en empresas legalmente constituidas.

Tal y como se expresó, el tema radica en que la consecución de clientes, no debe hacerse solo y exclusivamente por el subsidio familiar, sino por los servicios adicionales que deben tener todas las cajas en pos de un bienestar para el afiliado y sus familias.

Es así, como basado en la entrevista, es posible plantear que hay que llegar al afiliado (Cliente), a través de un marketing agresivo, o pull, es por ello que se maneja el marketing one to one, pero esto no es del todo suficiente para lograr captar ms clientes.

Surge entonces, en primera instancia el marketing relacional, como una primera estrategia que, a juicio de los autores deben diseñar las cajas. Por lo tanto, es interesante abordar la importancia del mix comunicacional, que obedece a un plan comunicacional de marketing, y que en realidad muchas cajas no lo tienen.

El consumidor actual se enfrenta a diario a una infinidad de mensajes orientados a provocar en él un efecto determinado. Estos mensajes, claramente construidos como publicitarios o disfrazados de información, persiguen al moderno consumidor en todas y cada una de sus facetas diarias: laborales, familiares, sociales y, por supuesto, personales. La televisión, la radio, el cine, los medios impresos, el correo, el or- denador, el teléfono e incluso el boca a oreja, son las bases desde las que las marcas tienden a construir los mensajes con los que pretenden llegar a ese inmenso colectivo que formamos los consumidores.

Sin embargo, la utilización de cada vez más medios para poder llegar hasta los sujetos están provocando el efecto contrario al deseado por las marcas; y es que, ante tal exceso de diferentes mensajes, el consumidor comienza a ser escéptico y no sólo no asimila, sino que cuestiona, la validez de los mensajes que recibe (Reinares \& Fernández, 2003).

Para el caso de las cajas de compensación, se es claro, que no pueden seguir trabajando bajo el amparo del marketing tradicional sino quieren deja de existir en el mercado, es por ello, que una buena base de marketing relacional estará diseñada a la medida de ellas, teniendo en cuenta los siguientes parámetros: El marketing relacional puede definirse como una estrategia de negocio o actividad que persigue la captura de un mayor "share of wallet" utilizando datos sobre clientes y el análisis de los mismos, con trasparencia en el razonamiento económico, y con el objetivo de adquirir, retener y desarrollar a los clientes rentables de una forma aún más rentable.

Para efectos de este artículo de investigación, el share of wallet, la cuota de cartera, que es como se conoce al share of wallet en español, ayuda a decidir si asignar recursos para ampliar la categoría de productos, conquistar clientes de otros competidores o incrementar la frecuencia de compra. Las cajas de compensación deben tener un objetivo claro con respecto a sus segmentos y donde invertir sobre la rentabilidad de los clientes que ya tiene y los potenciales. De esta manera es conveniente el marketing relacional para; Integrar todas las funciones de la caja; Uso expandido de tecnologías de la información. (Facebook, WhatsApp, Instagram, entre otros.); Visión global. Perspectiva de visión empresarial; Alianzas estratégicas y cooperación con marketing. Con los proveedores de la caja; Relaciones de largo plazo. Retención de afiliados; Socios.

En vez de afiliados, se trata de hacer a cada cliente (o pequeños grupos de clientes) actual o potencial la 
oferta que consiga maximizar su valor (en términos de tasa de éxito, ingresos, coste y duración), mediante la generación y explotación eficaz de la información que existe sobre él en la empresa. El punto central de este tipo de marketing consiste en retener clientes y venderles nuevos más rentables productos (servicios) muy adaptados a sus necesidades (cross-selling y up-selling).

Desde el punto de vista de los costes de la empresa suele resultar más rentable retener a los clientes existentes que empeñarse en captar clientes nuevos. El camino para retenerles es tenerles siempre satisfechos, ofreciendo en todo momento productos cada vez más a su medida que cubran sus necesidades, y para ello es preciso disponer de grandes cantidades de información. En resumen, los consumidores dejan de ser clientes para convertirse casi en auténticos socios de cada compañía (Reinares \& Fernández, 2003).

Es así como las cajas de compensación deben realizar esfuerzos para relacionarse con sus afiliados a través de las siguientes formas de comunicación; $\mathrm{Pu}-$ blicidad. A través de estrategias ATL y BTL, así mismo a través del marketing digital, esto con el fin de hacer marketing de atracción conseguir nuevos clientes; Marketing directo. One to one, para hacer relación de fidelización con los afiliados y sus familias; Marketing promocional. Acorde con el plan publicitario de comunicaciones de la empresa a través del IMC al que se refirió anteriormente; Animación. Consolidando estrategias de merchandising en las distintas sedes de las cajas; Realización de eventos. En algunos casos, los eventos serán patrocinados por la misma caja para buscar aumentar la fidelización de los afiliados existentes y atraer a los potenciales; Comunicación digital. A través de sus páginas web (que deben estar constantemente actualizadas), Twitter, Facebook, Instagram, YouTube, entre otros.

Además, debe contar con las siguientes herramientas de marketing; Minería de datos. Un database management, el cual debe estaré actualizado con respecto a sus afiliados y esta gestión de datos e información debe ser respaldada por un sistema de seguridad fiable. Esto conlleva a realizar una gestión de modelos predictivos para prospectar; Análisis clúster. En caso de que esta estrategia sea prioridad para las cajas, es un buen recurso para que posteriormente se presten los servicios demandados por los afiliados y sus familias. Se hace énfasis en la segmentación y microsegmentación, que deben ser propias de las cajas de compensación; Call center. La plataforma de gestión telefónica de cada caja. Esto no debe ser una tendencia de moda, sino una prioridad de la gerencia del servicio; El call center es un sistema de relación con los afiliados a través de la voz y no a través de máquinas contestadoras; Fulfillment. Teniendo en cuenta la importancia del manejo de la información, sobre todo el tema de habeas data.

En este sentido los empresarios tienen la opción de externalizar el proceso de Fulfillment y distribución o crear un departamento interno que se encargue de este proceso; Análisis de resultados. Una vez se realicen todas las actividades, se analizan todos los ítems para verificar los resultados a través de Kpis. La palabra KPI proviene de "Key Performance Indicators". Esto se traduce, ni más ni menos, como los "indicadores claves de desempeño". Es decir, aquellas variables, factores, unidades de medida, que consideramos "estratégicas" en nuestra empresa. Y que por ende influyen directamente en el "core business".

Todo esto se refleja en el tema principal de segmentos y microsegmentos, quienes finalmente son la razón de ser de las cajas, esto incluye a los clientes internos y a los stakeholders, quienes son todas aquellas personas, grupos y entidades que tienen intereses de cualquier tipo en una empresa y se ven afectados por sus actividades. Son interesados, directos o indirectos, en que la empresa funcione ya que en caso contrario les afectaría directamente.

Finalmente, para este tema de marketing relacional, se exponen 12 claves para que las cajas actúen en concordancia con sus afiliados. Estos son que todos los afiliados no son iguales. Teniendo en cuenta que los segmentos son diferentes, según Farné (2010) la clasificación de estos segmentos es: Dependientes; Pensionados; Voluntarios; Facultativos; Afiliados por fidelidad. Es necesario reconocer los momentos 
claves; Las promociones no fidelizan; Es necesario crear valor; La fidelización es sinónimo de complicidad; El proceso es el mensaje; La creatividad es de vital importancia; Conocer a profundidad al afiliado; Reconocer la importancia de la marca; Saber aprovechar los medios digitales; Conocer a los nuevos intermediarios; Conocer a fondo la logística.

El Marketing relacional para las cajas; Subsidios; Créditos; Educación; Ciencia y cultura; Recreación y deportes; Programas sociales; Agencia de empleo; Vivienda; Salud; Fomento empresarial; Esparcimiento; Bibliotecas; Otros: Supermercados y droguerías; Teniendo en cuenta esto, se establece el marketing social, correspondiente al marketing de las cajas de compensación, tal y como lo anota (Duque, 2016).

Implica definir estrategias que permitan un mayor acceso a los diferentes servicios que presta la Caja de Compensación Familiar, por parte de sus trabajadores afiliados, especialmente los que pertenecen a las categorías A y B, como se define desde el área de bienestar social de la Caja, es una tarea que implica la realización de actividades desde los siguientes enfoques; Hacer de los servicios de bajo costo o gratuitos una prioridad; En el caso del programa de vivienda tener ofertas financieras accesibles a los trabajadores, La colaboración activa y constante con socios internos y externos; Se incluye a proveedores que puedan proporcionar componentes esenciales a los programas. Es así, como dentro del marketing también debe incluir la responsabilidad social empresarial como uno de los pilares principales dentro de la organización (Del Río-Cortina, Cardona -Arbeláez \& Guacarí-Villalba, 2017).

Además, se recomienda aprovechar las ventajas de cada uno sin perder de vista los objetivos de la campaña. Para poner en marcha la estrategia de marketing de contenidos tienes a disposición; El blog de tu empresa; Posicionarte para un tema de forma orgánica; Crear contenido de valor para tus lectores; Ser conocido como experto en tu sector; Oportunidad de captar registros para ir creando una base de datos propia; Dar a conocer tu marca, producto o servicio; Las redes sociales.
Lo anterior implica establecer una relación directa con las empresas y sus trabajadores afiliados, de tal manera que las respuestas a las necesidades aporten no solo facilidad de acceso a los diferentes servicios del portafolio de la Caja, sino, a coadyuvar en el mejoramiento de los estándares del nivel de vida; facilitando así mismo el desarrollo de los principios corporativos de responsabilidad social a las empresas y el avance en sus índices de productividad; fruto de los nuevos indicadores de vida que posean sus empleados. Dada su misión vocacional, las Cajas de Compensación Familiar se han consolidado como gestores sociales y como una extensión de la responsabilidad social empresarial de sus empresas afiliadas. Es por esto, que las estrategias de marketing social tienen alta relevancia como estrategia de posicionamiento (Del Rio Cortina, Cardona-Arbeláez \& Simancas-Trujillo, 2017). 


\section{Conclusiones}

De acuerdo al análisis conceptual realizado sobre el nuevo marketing del siglo XXI, las cajas de compensación se ven en la imperiosa necesidad de reformarse ante un mercado cada día crece y se vuelve más competitivo y en competencia. Es así como los cambios de la dinámica del mercado hacen clientes, en este caso afiliados más exigentes. Los retos son mayores, cuando la gerencia moderna está obligada estratégicamente a trabajar con las Megatendencias del marketing.

Las estrategias identificadas como mayor impacto y que direccionarían a una gerencia moderna son: el marketing relacional, el marketing emocional y el reto de la gerencia del servicio, que para este caso recae en la dirección de la caja. Kotler (2005) enuncia los siguientes: que son aplicables a las cajas de compensación: Obtener mejores indicadores financieros acerca del impacto de los programas de marketing. En este caso son los programas de las mega tendencias; Hacer que el marketing sea el diseñador y el factor que impulse la estrategia de mercado de la compañía. A través de su departamento de marketing o de la gerencia del servicio, con énfasis en la tecnología; Enfrentar precios bajos o competidores que proporcionen una mejor calidad. La competencia es cada día más fuerte, mas no porque existan más cajas, sino por el número creciente de la demanda para pocas ofertas.

Además, las Megatendencias que acompañan a esta investigación son entre otras: El marketing de experiencias, el marketing digital, productos y servicios personalizados, concentración, posicionamiento y diferenciación, creatividad e innovación.

\section{Referencias}

Aaker, D.A., Day, G. S., \& Kumar, V.(2001). Investigación demercados. México: Limusa.

Duque, D. (2016). El marketing social como metodología diferencialen la estrategia de negocio de las organizaciones (Tesis de maestría). Universidad nacional de Colombia, Sede Manizales, Colombia.

Del Río Cortina, J., Cardona-Arbeláez, D., \& Simancas-Trujillo, R. (2017). Propuesta de un modelo teórico de branding para el posicionamiento de la marca universitaria. Espacios, 38(53). https://doi.org/https://hdl.handle.net/20.500.12585/8968

Del Río-Cortina, J. L., Cardona-Arbeláez, D., \& Guacarí-Villalba, A. (2017). Responsabilidad social empresarial y construcción de la marca: una nueva mirada a las estrategias de gestión. Revista de Investigación, Desarrollo e Innovación, 8(1), 49. https://doi. org/10.19053/20278306.v8.nl.2017.7370

Farné, S. (2010). Boletín del observatorio del mercado de trabajo y la seguridad social. La parafiscalidad laboral en Colombia. https://www.uexternado.edu.co/wp-content/uploads/2017/01/ boletin 12.pdf

Kotler, P. (2005). Las preguntas más frecuentes sobre marketing. Cali, Colombia: Editorial Norma.

Reinares, P., \& Fernández, S. (2003). Marketing relacional, un nuevo enfoque para la seducción y fidelización del cliente. Madrid, España: Pearson Educación.

Ries, A., Trout, J. (1993). Las 22 leyes inmutables de marketing. México: Mc Graw Hill.

Oconnor, L., Zaldívar,A., \& Hernández, E. (2011). Un acercamiento al estudio de la integración de métodos teóricos de la investigación científica. Revista Universitaria Arbitrada de Investigación y Diálogo Académico,7(1), 142-57.

Orozco, J. A. (1999). Investigación de mercados. Concepto y práctica. Cali, Colombia: Grupo Editorial Norma.

Zeithaml, V., \& Bitner, M. (2001). Marketing de servicios un enfoque de integración del cliente a la empresa. México: Mc Graw Hill. 